\title{
Maximization of Left Atrial Information Through the Optimization of ECG Lead Systems
}

\author{
Axel Loewe ${ }^{1}$, Sebastian Debatin $^{1}$, Gustavo Lenis $^{1}$, Olaf Dössel $^{1}$ \\ ${ }^{1}$ Institute of Biomedical Engineering, Karlsruhe Institute of Technology (KIT), Karlsruhe, Germany
}

\begin{abstract}
Atrial fibrillation and atrial flutter are the most common atrial arrhythmias placing a heavy burden on patients and posing a challenge on healthcare systems. If patients at risk to develop atrial arrhythmias can be identified at an early stage, the arrhythmia incidence can be lowered by implementing appropriate strategies to mitigate the risks. Diagnostic methods based on the ECG are ideal risk markers due to their noninvasiveness and omnipresence. The left atrium (LA) plays a major role in the initiation and perpetuation of atrial reentry arrhythmias. However, the LA is not well represented in the P-wave derived through standard ECG leads. Here, we optimize ECG leads to maximize LA information content. Towards this end, we used a cohort of eight personalized computational models providing the unique opportunity to separate LA and right atrial (RA) contributions to the $P$-wave, which is not feasible in vivo. The location of maximum P-wave signal energy was located on the center of the chest for all subjects with marked overlap between regions of maximum $L A$ and $R A$ $P$-wave amplitude. The regions of highest ratio between $L A$ and RA signal energy differed between patients. However, a region with LA signal energy being higher than that of the $R A$ and providing a sufficiently large absolute P-wave signal energy was identified at the lower left quadrant of the back consistently across most subjects of the cohort. Optimized linear combinations of standard 12-lead signals (considering the eight independent leads) yielded comparably good results amplifying LA information by more than one order of magnitude.

Our newly proposed electrode positions on the back as well as selected combinations of standard ECG signals improve the LA information content considerably. By using these, more relevant diagnostic information regarding anatomical and electrophysiological properties of the LA can be derived in future.
\end{abstract}

\section{Introduction}

The ECG has great potential as a method to screen patients for their risk of cardiac diseases due to the fact that it is non-invasively acquired in routine examinations, cheap to obtain, and available almost everywhere. A cardiac disease posing a particular burden is atrial fibrillation (AF) with a prevalence of $2-3 \%$ in the general population and severe consequences like stroke and increased mortality. Considering these facts, it is not surprising that AF risk stratification based on the P-wave is clinically desirable $[1,2]$.

The left atrium (LA) plays a crucial role in the initiation and perpetuation of $\mathrm{AF}$ as both the substrate and the triggers driving $\mathrm{AF}$ are mostly located in the LA. Therefore, the maximization of the LA information in the $\mathrm{P}$-wave is of paramount importance to be as predictive as possible for atrial fibrillation risk. However, previous work of us and others $[3,4]$ has shown that the contribution of the LA to the standard ECG leads is limited compared to the contribution of the right atrium (RA). In this work, we propose a location for a nonstandard ECG lead maximizing left atrial information content as well as a linear combination of standard 12lead signals.

\section{Methods}

We comuputed atrial ECGs using a cohort of eight anatomically personalized realistic models and separated LA and RA contributions as described and validated before [4,5]. In brief, biatrial volumetric hexahedral models were constructed from imaging data and augmented with myocyte orientation, interatrial connections, and heterogeneous tissue labels using a rulebased algorithm [6,7]. Interatrial conduction was possible via Bachmann's bundle, two posterior connections, and via the coronary sinus. Cellular electrophysiology was represented by a heterogeneous version of the Courtemanche et al. membrane model with spatially varying maximum conductivities of $\mathrm{I}_{\text {to }}$ and $\mathrm{I}_{\mathrm{CaL}}[4,8]$. Anisotropic excitation propagation was computed using the monodomain solver acCELLerate [9] with conductivity values as in [4]. Extracellular potentials within the tetrahedral torso domain due to the spatial gradient of the transmembrane voltage were computed according to bidomain theory [5]. To separate RA and LA contributions, the sources $\nabla V_{m}$ were masked and set to zero in the other atrium during the forward calculation of electrical potentials to mute this atrium. 
The resulting body surface potential maps were referenced to Wilson's Central Terminal and the energy of the unipolar P-wave signal was calculated for each electrode i:

$$
P_{i}=\int_{P_{\text {begin }}}^{P_{\text {end }}} U_{i}^{2}(t) d t .
$$

Besides evaluating the whole torso surface to identify optimal locations for additional electrodes to maximize LA information content, we aimed to leverage the information contained in the standard 12-lead ECG. Towards this end, we optimized coefficients of linear combinations of the eight linearly independent standard ECG leads (I, II, V1-V6). The mixing vector was determined by considering the optimization as a generalized eigenvalue problem:

$$
C_{L A} V=C_{R A} V D \text {, }
$$

with $C_{L A}$ and $C_{R A}$ being the correlation matrices of the Pwave signals from the respective atria, $\mathrm{V}$ being a matrix of eigenvectors, and $\mathrm{D}$ being a diagonal matrix holding the eigenvalues. The column vector of $\mathrm{V}$ corresponding to the largest eigenvalue holds the coefficients maximizing the left-to-right atrial signal energy ratio (LRR) scaled by the largest eigenvalue. The linear combination of leads was optimized for each subject individually as well as across all eight subjects simultaneously. In addition to maximizing LRR, we computed the mixing vector maximizing the difference of the energies of the signals $\left(\mathrm{P}_{\mathrm{LA}}-\mathrm{P}_{\mathrm{RA}}\right)$.

\section{Results}

Figure 1 shows the spatial distribution of the P-wave signal energy stemming from both atria normalized to the highest energy value for subject \#2. The highest signal energy was found on the superior part of the chest laterally centered. When distinguishing LA (Figure $2 \mathrm{~A}+\mathrm{B}$ ) and RA (Figure $2 \mathrm{C}+\mathrm{D}$ ) sources, the energy maxima of the LA signal were located more inferior, particularly on the back. Areas with high RA signal energy covered the superior central and right parts of the chest and the back.

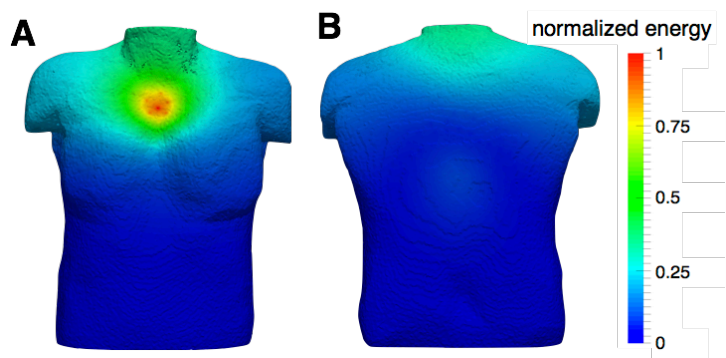

Figure 1. Spatial distribution of the P-wave energy stemming from both atria for subject \#2. Anterior (A) and posterior (B) aspect. Values are normalized to the maximum.
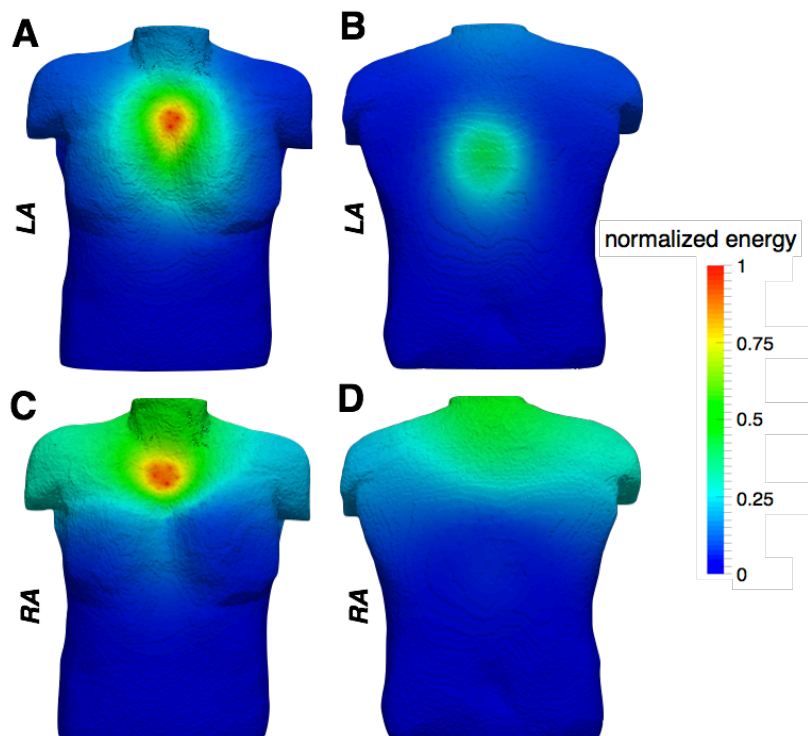

Figure 2. Spatial distribution of the P-wave energy stemming from each atrium separately for subject $\# 2$. Values are normalized to the maximum of each atrium.
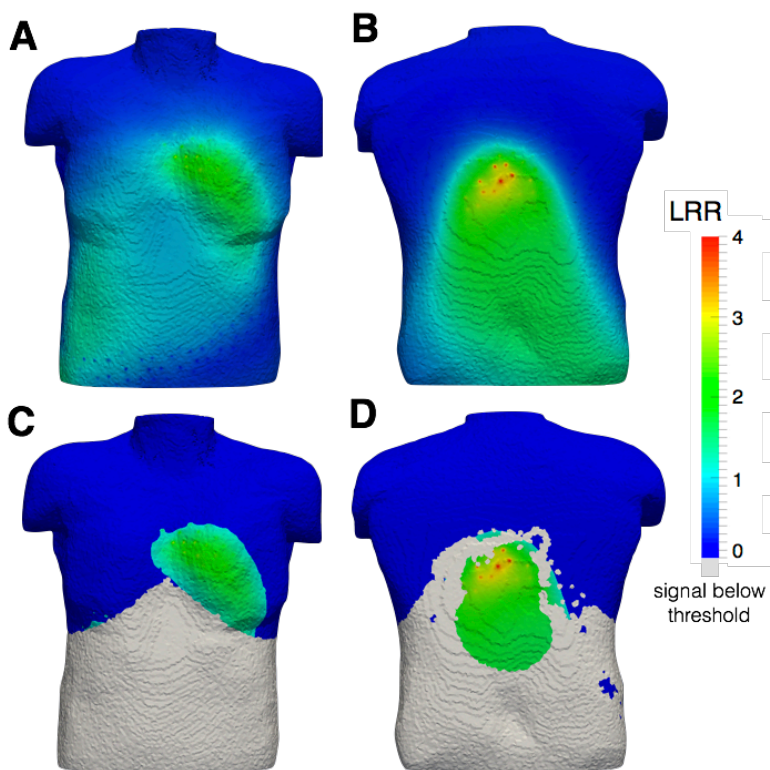

Figure 3. Left to right atrial P-wave energy ratio (LRR) for subject \#2 (A+B). In $\mathrm{C}+\mathrm{D}$, electrodes with a root mean square amplitude below $28.6 \mu \mathrm{V}$ were neglected.

To obtain a measure for the relative LA information content, LRR was calculated for each electrode (Figure $3 \mathrm{~A}+\mathrm{B})$. As LRR values can occur at rather small LA energies when the RA energy diminishes, we posed an additional condition of a minimal root mean square amplitude of $28.6 \mu \mathrm{V}$ during the $\mathrm{P}$-wave (Figure $3 \mathrm{C}+\mathrm{D}$ ). This threshold corresponds to a signal-to-noise ratio of $4.86 \mathrm{~dB}$ when averaging over $10 \mathrm{P}$-waves and assuming an average noise amplitude of $50 \mu \mathrm{V}$. 


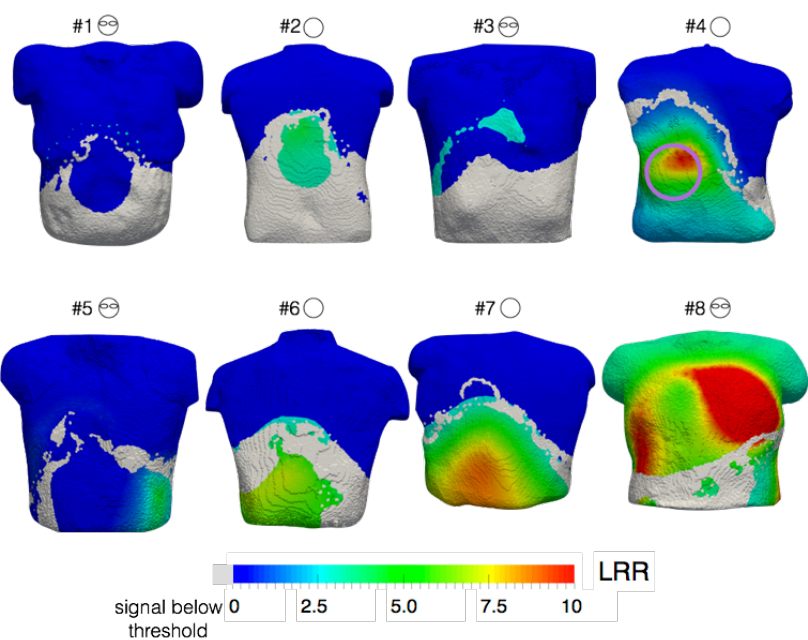

Figure 4. Left to right atrial P-wave energy ratios (LRR) for all eight subjects. The aspect (anterior/posterior) exhibiting the highest LRR is shown. Icons next to the subject index indicate the aspect. Electrodes with a root mean square amplitude below $28.6 \mu \mathrm{V}$ were neglected. The circular region indicated for subject \#4 was identified as the optimum electrode location for the whole cohort of eight models.

On the back of subject \#2, the highest LRR was observed laterally centered and oriented towards the inferior part (Figure 3B). On the chest, a circular structure around the center exhibited the highest LRR with the maximum values to the upper left (Figure 3A). When requiring a minimum root mean square signal amplitude, the inferior part of the torso could not be considered (Figure $3 \mathrm{C}+\mathrm{D}$ ).

The LRR patterns as well as the regions exceeding the amplitude threshold showed considerable inter-individual variability (Table 1, Figure 4). The superior chest and back showed consistently low LRR values while the inferior part of the chest did not reach the amplitude threshold. An exception with generally elevated LRR values up to 56.77 was observed for subject $\# 8$, which was the only subject with a history of $\mathrm{AF}$ and a significantly enlarged left atrium (136ml compared to $27-$ $87 \mathrm{ml}$ for the other subjects) [5]. Considering the LRR and signal amplitude patterns of all eight subjects in the cohort, a common region in the lower left part of the back was identified as a promising target region to place an additional ECG electrode maximizing LA information content (Figure 4, subject \#4). Within this region, the maximum LRR across all subjects was 0.72-21.7 and the minimum LRR ranged between 0.31 and 15.81 (Table 1).

In a next step, we constrained the information to signals derived from linear combinations of the standard 12-lead ECG. By finding the ideal linear combination for each subject individually, LRR values between 93 and 580 could be obtained compared to values between 0.02 and 8.38 for the standard leads. When optimizing for a common mixing vector, i.e. requiring the same linear combination of leads for all subjects, LRR values declined to 0.4-116. However, the same challenge regarding absolute signal amplitude and its relation to noise as discussed above holds for these signals. Therefore, we maximized the difference of the energies of the $\mathrm{P}$-wave signals $\left(\mathrm{P}_{\mathrm{LA}}-\mathrm{P}_{\mathrm{RA}}\right)$ favoring higher signal amplitudes. The LRR for the signals projected onto this basis ranged from 0.47 to 10.8 (Table 1). The optimal coefficients for leads I-II and V1-V6 for the latter scenario were $0.17,0.38,-0.34,-0.64,-0.42,-0.15,0.06$, and 0.31 , respectively.

\section{Discussion}

In this study, we aimed to maximize LA information content in the body surface P-wave by i) identifying the best location for a unipolar ECG recording and ii) by providing an optimal linear combination of standard ECG leads. The LRR could be increased to values significantly above 1 using both an individually optimized electrode location, as well as an individually optimized mixing vector. However, for translational perspectives, a common method for all patients (or at least for a group of patients) is required if no model is to be built for each

Table 1. Left-to-right atrial P-wave energy ratios (LRR) for the eight subjects of the model cohort. The first row represents the individually best unipolar electrode on the whole torso for each model, rows 2-4 represent the common target region in the lower left part of the back (Figure 4). The last 3 rows use only information from a standard 12-lead ECG. Mixing vectors were optimized to maximize LRR for each subject individually or for all subjects simultaneously ("common") and to maximize the energy of the LA-RA difference signal ("diff") for all subjects simultaneously.

\begin{tabular}{|c|c|c|c|c|c|c|c|c|}
\hline Subject & $\# 1$ & $\# 2$ & $\# 3$ & $\# 4$ & $\# 5$ & $\# 6$ & $\# 7$ & $\# 8$ \\
\hline $\mathrm{LRR}_{\max }$ whole torso & 2.75 & 3.97 & 2.04 & 10.7 & 5.00 & 6.89 & 8.83 & 56.8 \\
\hline $\mathrm{LRR}_{\max }$ target region & 0.91 & 3.97 & 0.72 & 10.7 & 0.46 & 4.83 & 7.40 & 21.7 \\
\hline $\mathrm{LRR}_{\text {mean }}$ target region & 0.73 & 2.71 & 0.60 & 8.29 & 0.38 & 4.42 & 6.21 & 16.7 \\
\hline $\mathrm{LRR}_{\min }$ target region & 0.48 & 2.00 & 0.48 & 6.90 & 0.31 & 1.95 & 4.96 & 15.8 \\
\hline LRR 12-lead $\mathrm{max}_{\max }$ individual & 100 & 543 & 186 & 462 & 93.1 & 328 & 580 & 569 \\
\hline LRR 12-lead $\max$ common & 3.21 & 0.40 & 22.1 & 33.7 & 5.22 & 14.6 & 32.3 & 116 \\
\hline LRR 12-lead max $_{\max }$ comon, diff. & 1.02 & 1.53 & 2.09 & 3.08 & 0.47 & 2.30 & 3.88 & 10.8 \\
\hline
\end{tabular}


Patient. When aiming for a common method, LRR was reduced but remained above 1 for most models and increases LRR by a least one order of magnitude for all models. Comparing unipolar signals from the optimized location in the lower left part of the back with a linear combination of standard 12-lead signals, LRR values were in a similar range with a tendency towards higher LRR for linear combinations. However, the fundamental difference between unipolar and bipolar ECG signals should not be neglected and leaves room for further improvement of optimized electrode locations. Nevertheless, considering practical issues like number of hardware channels available, required training and persuasion for new electrode positions, possibility of doing retrospective analyses, etc., an optimized linear combination of standard ECG leads appears to be the most promising way to maximize LA information content in the P-wave.

The fact that only anatomical variability and not electrophysiological interindividual variability was accounted for has to be considered a limitation of this study. Moreover, the cohort of eight subjects comprised mostly structurally healthy atria [5] with the exception of subject \#8, who had a history of documented AF. This should be considered when extrapolating our findings to diseased atria. While the size of the cohort is small compared to clinical studies, we are confident that it covers a good share of the anatomical variability of healthy atria. In combination with the perfectly controlled conditions inherent to in silico experiments, we are confident that the conclusions drawn here are representative for the general population. The interindividual variability in the results highlights the importance of using a cohort of models instead of a single anatomical model.

In conclusion, we presented methods to maximize LA information content in the ECG signal. Particularly optimized linear combinations of standard 12-lead signals amplified LRR by more than one order of magnitude and can in future be used to optimize diagnostic tools. Important examples are ECG markers as surrogate measures to assess LA anatomy and function [10-12]. The sensitivity and specificity of classifiers derived from these markers regarding risk stratification and disease classification are currently not satisfactory. By maximizing the LA information content in the input signal using the methods presented in this paper, their performance could be boosted.

\section{Acknowledgments}

We gratefully acknowledge financial support by the Deutsche Forschungsgemeinschaft (DFG) through CRC 1173.

\section{References}

[1] Magnani JW. Risk assessment for atrial fibrillation: Enter the P-wave. Heart Rhythm. 2015;12:1896-7.

[2] Altunkeser BB, Ozdemir K, Gok H, et al. Can P wave parameters obtained from 12-lead surface electrocardiogram be a predictor for atrial fibrillation in patients who have structural heart disease? Angiology. 2003;54:475-9.

[3] Jacquemet V. Modeling left and right atrial contributions to the ECG: A dipole-current source approach. Comput Biol Med. 2015;65:192-9.

[4] Loewe A, Krueger MW, Platonov PG, et al. Left and Right Atrial Contribution to the P-wave in Realistic Computational Models. In: Functional Imaging and Modeling of the Heart 2015, Lecture Notes in Computer Science. 2015;9126:439-47.

[5] Loewe A, Krueger MW, Holmqvist F, et al. Influence of the earliest right atrial activation site and its proximity to interatrial connections on $\mathrm{P}$ wave morphology. Europace. 2016;18:iv35-iv43.

[6] Wachter A, Loewe A, Krueger MW, et al. Mesh structure-independent modeling of patientspecific atrial fiber orientation. Curr Dir Biomed Eng. 2015;1:409-12.

[7] Loewe A. Modeling human atrial pathoelectrophysiology from ion channels to ECG. Karlsruhe: KIT Scientific Publishing; 2016.

[8] Krueger MW, Dorn A, Keller DUJ, et al. In-silico modeling of atrial repolarization in normal and atrial fibrillation remodeled state. Med Biol Eng Comput. 2013;51:1105-19.

[9] Seemann G, Sachse FB, Karl M, et al. Framework for Modular, Flexible and Efficient Solving the Cardiac Bidomain Equations Using PETSc. Prog Ind Math (ECMI 2008). 2010;15:363-9.

[10] Wagner GS, Macfarlane P, Wellens $H$, et al. AHA/ACCF/HRS recommendations for the standardization and interpretation of the electrocardiogram: part VI: acute ischemia/infarction. J Am Coll Cardiol. 2009;53:1003-11.

[11] Loewe A, Andlauer R, Platonov PG, et al. Left atrial hypertrophy increases $\mathrm{P}$-wave terminal force through amplitude but not duration. In: Computing in Cardiology. 2016;43:1-4.

[12] Platonov PG. P-wave morphology: Underlying mechanisms and clinical implications. Ann Noninvasive Electrocardiol. 2012;17:161-9.

Address for correspondence:

Axel Loewe, PhD; Institute of Biomedical Engineering,

Karlsruhe Institute of Technology (KIT)

Fritz-Haber-Weg 1, 76131 Karlsruhe, Germany

publications@ibt.kit.edu 